\title{
Tracking cancer lesions on surgical samples of gastric cancer by artificial intelligent algorithms
}

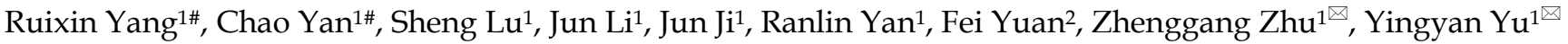 \\ 1. Department of General Surgery of Ruijin Hospital, Shanghai Institute of Digestive Surgery, and Shanghai Key Laboratory for Gastric Neoplasms, Shanghai \\ Jiao Tong University School of Medicine, 200025, Shanghai, China. \\ 2. Department of Pathology of Ruijin Hospital, Shanghai Jiao Tong University School of Medicine, 200025, Shanghai, China.
}

\#These authors contributed equally to this work.

$\triangle$ Corresponding authors: Yingyan Yu, Tel: (+86)21-34187336; Fax: (+86)21-64333548; E-mail: yingyan3y@sjtu.edu.cn; Zhenggang Zhu, Tel: (+86)21-34187336; Fax: (+86)21-64333548; E-mail: zzg1954@hotmail.com.

(c) The author(s). This is an open access article distributed under the terms of the Creative Commons Attribution License (https://creativecommons.org/licenses/by/4.0/). See http://ivyspring.com/terms for full terms and conditions.

Received: 2021.06.15; Accepted: 2021.08.29; Published: 2021.09.03

\begin{abstract}
To quickly locate cancer lesions, especially suspected metastatic lesions after gastrectomy, Al algorithms of object detection and semantic segmentation were established. A total of 509 macroscopic images from 381 patients were collected. The RFB-SSD object detection algorithm and ResNet50-PSPNet semantic segmentation algorithm were used. Another 57 macroscopic images from 48 patients were collected for prospective verification. We used $\mathrm{mAP}$ as the metrics of object detection. The best mAP was $95.90 \%$ with an average of $89.89 \%$ in the test set. The mAP reached $92.60 \%$ in validation set. We used mloU for evaluation of semantic segmentation. The best mloU was $80.97 \%$ with an average of $79.26 \%$ in the test set. In addition, 81 out of $92(88.04 \%)$ gastric specimens were accurately predicted for the cancer lesion located at the serosa by ResNet50-PSPNet semantic segmentation model. The positive rate and accuracy of $\mathrm{Al}$ prediction were different based on cancer invasive depth. The metastatic lymph nodes were predicted in 24 cases by semantic segmentation model. Among them, 18 cases were confirmed by pathology. The predictive accuracy was $\mathbf{7 5 . 0 0 \%}$. Our well-trained Al algorithms effectively identified the subtle features of gastric cancer in resected specimens that may be missed by naked eyes. Taken together, Al algorithms could assist clinical doctors quickly locating cancer lesions and improve their work efficiency.
\end{abstract}

Key words: Artificial Intelligence; Object Detection; Semantic Segmentation; Gastric Cancer; Macroscopic Images

\section{Introduction}

Gastric cancer is one of the most common gastrointestinal cancers with the fifth incidence and the second mortality worldwide [1]. The rapid and accurate diagnosis of gastric cancer will improve the treatment outcome of patients. Gastrectomy is a key therapeutic measure for gastric cancer. After gastrectomy, pathologists will examine and report the cancer histology and possible metastatic status. This procedure takes a lot of time, especially when the cancer is at its early stage. Therefore, quickly locating the cancer lesions, especially suspected metastatic lesions will improve the work efficiency. In this study, artificial intelligence (AI) algorithms of object detection and semantic segmentation were used to identify gastric cancer in surgical specimens.
AI progresses quickly in the field of medical images [2]. Convolutional neural network $(\mathrm{CNN})$ is a deep learning network for computer vision analysis [3]. It is proper for analyzing medical images including image classification, object detection and semantic segmentation. Image classification using CNN for gastric mucosal lesions has achieved progression in our previous study [4]. Using the excellent ability of CNN for feature extraction, more robust algorithms of object detection and semantic segmentation are being developed [5]. There are a variety of $\mathrm{CNN}$ models with different precision and training speed including VGG, Inception, ResNet, MobileNet, Xception, EfficientNet, and so on [6-9]. 
To identify the cancer lesions and suspected metastatic lymph nodes accurately, we constructed the object detection and semantic segmentation algorithms with CNN backbones. The AI algorithms could accurately recognize the gastric cancer lesions in macroscopic images, and also predict metastatic lesions in surrounding lymph nodes. To our knowledge, this is the first report that AI technology could be used for assisting cancer lesion recognition in post-operative specimens.

\section{Methods}

\section{Model construction and image labeling}

All macroscopic images of stomach were obtained after gastrectomy as JPG format. The inclusion criteria was the specimens come from surgical resection of confirmed gastric cancer. The exclusion criteria included low resolution images, unclear location or obvious bleeding. A total of 509 macroscopic images from 381 patients of gastric cancer were included in the object detection and semantic segmentation analysis. Among them, 460 out of 509 (90\%) images from 347 patients were used as the training set, and 49 out of $509(10 \%)$ images from 34 patients as test set. All images were obtained from the Department of General Surgery, Ruijin Hospital of Shanghai Jiaotong University School of Medicine. This study was approved by the Research Ethics Committee of Shanghai Ruijin hospital. The written informed consents were signed by all patients.

Macroscopic images were labeled by surgical experts (YC and YR) with LabelImg (an open-source platform for object detection image annotations; https://github.com/tzutalin/labelImg), and Labelme (an open-source platform for semantic segmentation image annotations; https://github.com/wkentaro/ labelme). The key steps included inputting of macroscopic image, manual labeling and image outputting. In object detection analysis, the macroscopic images were manually labeled with priori boxes, and then saved with Human-readable Extensible Markup Language (XML) format. In semantic segmentation analysis, macroscopic images were manually labeled and saved with boundary and JavaScript Object Notation (JSON) format. The subsequent analysis would extract labeling information from JSON files to transfer original images into pixels images with red lesion $(255,0,0)$ and black background $(0,0,0)$.

\section{Prospective validation set}

The prospective validation set was an independent set for evaluating model performance to ensure the generalization ability. All images were saved in JPG format. The exclusion criteria were the same as that of the training set. Finally, 57 images from 48 patients were included. Images in the prospective validation set did not overlap with that in the training set.

\section{The serosal appearance analysis}

To analyze the involving status of gastric serosa, we collected 92 resected stomach specimens from advanced gastric cancer. These images were used for predicting serosal invasion at the status of no incision of stomach.

\section{K-fold cross validation}

K-fold cross validation was used according to previous reports for getting better training efficiency when the primary data was limited [10, 11]. After K times training, the best model weight was defined according to the evaluation in each fold using the test set.

\section{Computational requirements and algorithms}

The study was performed using the hardware Intel Core i7-10750H CPU, 16G RAM, NVIDIA GeForce RTX 2060 and the 6G VRAM.VOC2007 (Visual Object Classes2007) was used for transfer learning images [12,13]. By preliminary tests on 12 object detection models (MobileNet-SSD, VGG16SSD, RFB-SSD, YOLO-V3, YOLO-V4, YOLO-V4-tiny, RetinaNet, M2det, CenterNet, EfficientDetD0 and D1, and Faster RCNN) [8, 9, 14-17] and 9 semantic segmentation models (MobileNet-Unet, VGG16-Unet, MobileNet-PSPNet, ResNet50-PSPNet, CENet, FCN, CFPNet, DCUNet and ICNet) [18-20], the RFB-SSD object detection and PSPNet semantic segmentation revealed the best performance, and were used in this study. All computation was run on the Google's Tensorflow and Keras deep learning framework based on python language [21].

\section{Construction of RFB-SSD object detection model}

The Receptive Field Block-Single Shot MultiBox Detector (RFB-SSD) model was the integration of RFB module with VGG16, and it increased the ability of feature extraction and object detection[22]. To obtain the best performance for detecting gastric cancer in macroscopic images, RFB-SSD model was dynamically trained by 10 -fold cross validation with the maximum epochs as 100 . In the training process, Adam was used to optimize convergence speed. The MultiboxLoss was used to reduce the imbalance of the positive and negative samples[14]. The input size of training and evaluating images were $300 \times 300 \times 3$ pixels. The evaluation parameters of object detection include mean average precision (mAP), log-average miss-rate (LAMR), precision, recall and F1. 


\section{Construction of ResNet50-PSPNet semantic segmentation model}

The pyramid pooling module was introduced into PSPNet semantic segmentation with multiple convolutional kernels of different size for construction of pooling feature pyramids [23]. The key steps include ResNet50 preliminary feature extraction and PSPNet enhanced feature extraction. To obtain the best performance, ResNet50-PSPNet model was dynamically trained by 10 -fold cross validation with the maximum epochs as 100. In the training process, Adam was used to optimize semantic segmentation. The dice loss was used as the loss function to reduce the imbalance of the positive and negative samples [24]. The input size of the training and evaluating images were $273 \times 273 \times 3$ pixels. The evaluation parameters of semantic segmentation include intersection over union per class (class IoU) and mean IoU (mIoU).

\section{Statistical analysis}

In the object detection algorithm, the evaluation parameters include $\mathrm{mAP}$, LAMR, precision, recall and F1. In the semantic segmentation algorithm, the evaluation parameters include IoU and mIoU. The difference of different algorithms was compared by Wilcoxon paired signed-rank test with $p<0.05$ as statistical significance. All tests were bilateral. The Prism (8.0, GraphPad) was used for statistical analysis and plotting.

\section{Results}

\section{The performance of object detection algorithm}

Among the 12 selected object detection algorithms, RFB-SSD algorithm (Figure 1A) revealed the highest $\mathrm{mAP}$ of $96.36 \%$, and the lowest LAMR of 0.000001 with $90 \%$ precision, $90 \%$ recall and F1 0.9 (Table 1). So, RFB-SSD was used for the subsequent analysis. Based on the 10-fold cross validation in the training set (Table S1), the mean $\mathrm{mAP}$ reached $89.89 \%$ (95\% CI, 87.49\%-92.29\%), and the LAMR reached 0.16 (95\% CI, 0.12-0.20). At the 0.5 score threshold, the precision was $88.21 \%$ (95\% CI, $85.92 \%-90.49 \%)$, the recall was $88.37 \%$ (95\% CI, 85.79\%-90.95\%), and the F1 was 0.89 (95\% CI, 0.86-0.91). In the test set, the best prediction accuracy reached $91.84 \%$ with the mAP of 95.90\% (Figure 1B, left up). The LAMR reached 0.07 at the fold four steps. At the 0.5 score threshold, the precision was $92.16 \%$ (Figure 1B, right up), the recall was $95.92 \%$ (Figure 1B, left down), and the F1 was 0.94 (Figure 1B, right down).

In the test set, the prediction accuracy for gastric cancer was $91.84 \%$ and the false negative rate (FNR) was $8.16 \%$. For the true-positive lesions, the confidence and classification result presented on the top of the yellow boxes were of a high confidence $(0.97 \pm 0.07)$ (Figure 1C). Three lesions fell into the false-positive category with a low confidence $(0.65 \pm 0.05)$ (Figure 1D). The confidence between the true-positive and false-positive groups were significantly different $(p<0.0001)$. We further analyzed the reason of false-positive cases and found that the predictive box of one case was overlapped with the rugae of the greater curvature of the stomach, which mimicked ulcerative lesion (Figure 1D, left). In another case, the predictive box overlapped with a surgical metal clip, which interfered with the AI analysis (Figure 1D, mid). The last false-positive case was the gastric stump with distorted anatomical structure, which was rarely learnt by AI during the training procedure (Figure 1D, right). In addition, the AI failed for predicting lesions in two cases, one was a diffuse type lesion (type Borrmann IV) lacking obvious lesion boundary and the other was from the gastric stump with distorted anatomical structure, which was rarely learnt by AI during the training procedure. The results showed that the RFB-SSD object detection algorithm performed well in positioning cancer lesions in resected specimens. This algorithm revealed fast speed and high accuracy.

Table 1. The performance of several object detection models

\begin{tabular}{|c|c|c|c|c|c|c|c|}
\hline Models & Feature extraction network & Object detection & mAP & LAMR & F1 & Precision & Recall \\
\hline MobileNet-SSD & MobileNet & SSD & $85.73 \%$ & $2.6 \times 10^{-1}$ & 0.76 & $93.55 \%$ & $64.44 \%$ \\
\hline VGG16-SSD & VGG16 & SSD & $77.99 \%$ & $3.5 \times 10^{-1}$ & 0.74 & $77.78 \%$ & $70.00 \%$ \\
\hline YOLO-V3 & Darknet53 & YOLO-V3 & $74.90 \%$ & $3.1 \times 10^{-1}$ & 0.75 & $100.00 \%$ & $60.00 \%$ \\
\hline YOLO-V4 & CSPDarknet53 & YOLO-V4 & $85.36 \%$ & $2.4 \times 10^{-1}$ & 0.88 & $97.22 \%$ & $79.55 \%$ \\
\hline YOLO-V4-tiny & CSPDarknet53 & YOLO-V4-tiny & $81.00 \%$ & $2.9 \times 10^{-1}$ & 0.75 & $100.00 \%$ & $60.00 \%$ \\
\hline RFB-SSD & VGG-RFB & SSD & $96.36 \%$ & $2.9 \times 10^{-6}$ & 0.90 & $90.00 \%$ & $90.00 \%$ \\
\hline Retinanet & ResNet50 & Retinanet & $89.28 \%$ & $2.9 \times 10^{-3}$ & 0.86 & $81.82 \%$ & $90.00 \%$ \\
\hline M2det & VGG16 & M2det & $71.54 \%$ & $3.0 \times 10^{-1}$ & 0.82 & $100.00 \%$ & $70.00 \%$ \\
\hline CenterNet & ResNet50 & CenterNet & $73.47 \%$ & $3.6 \times 10^{-1}$ & 0.63 & $95.45 \%$ & $46.67 \%$ \\
\hline EfficientDet D0 & EfficientNetD0 & EfficientDet & $94.08 \%$ & $1.2 \times 10^{-1}$ & 0.85 & $90.00 \%$ & $80.00 \%$ \\
\hline EfficientDet D1 & EfficientNetD1 & EfficientDet & $94.34 \%$ & $1.2 \times 10^{-1}$ & 0.86 & $90.24 \%$ & $82.22 \%$ \\
\hline Faster RCNN & ResNet50 & Faster RCNN & $88.18 \%$ & $2.1 \times 10^{-1}$ & 0.72 & $60.00 \%$ & $90.00 \%$ \\
\hline
\end{tabular}


A

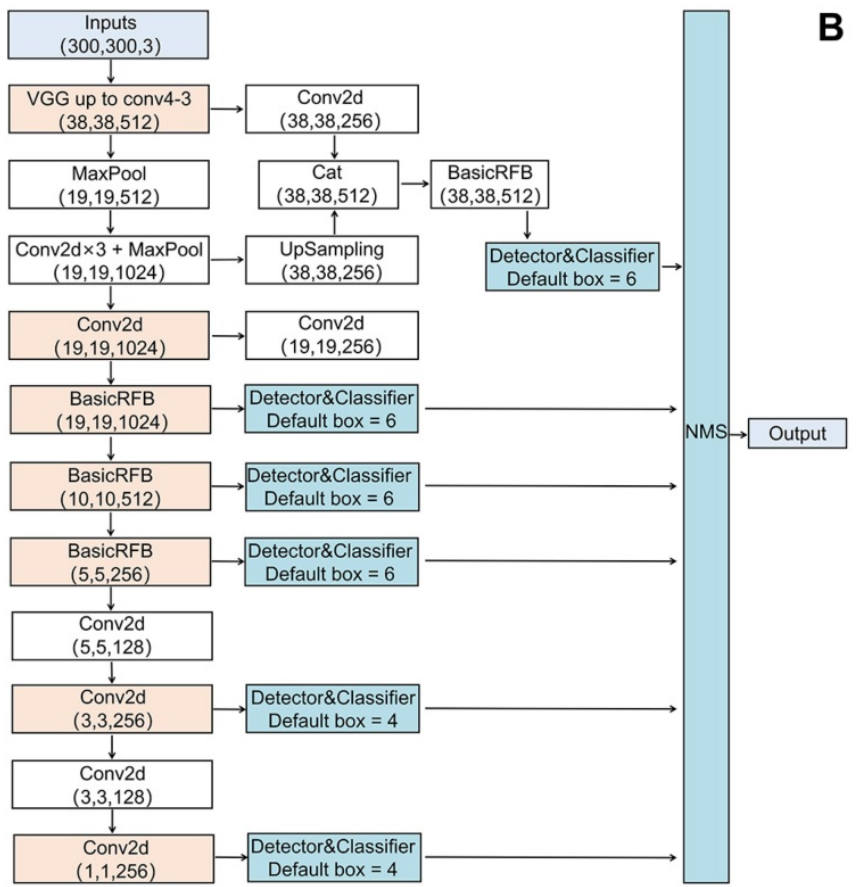

C

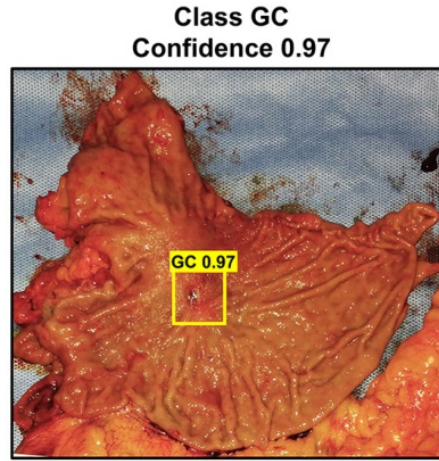

D

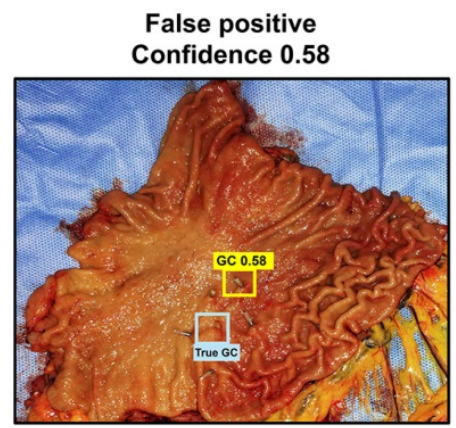

B
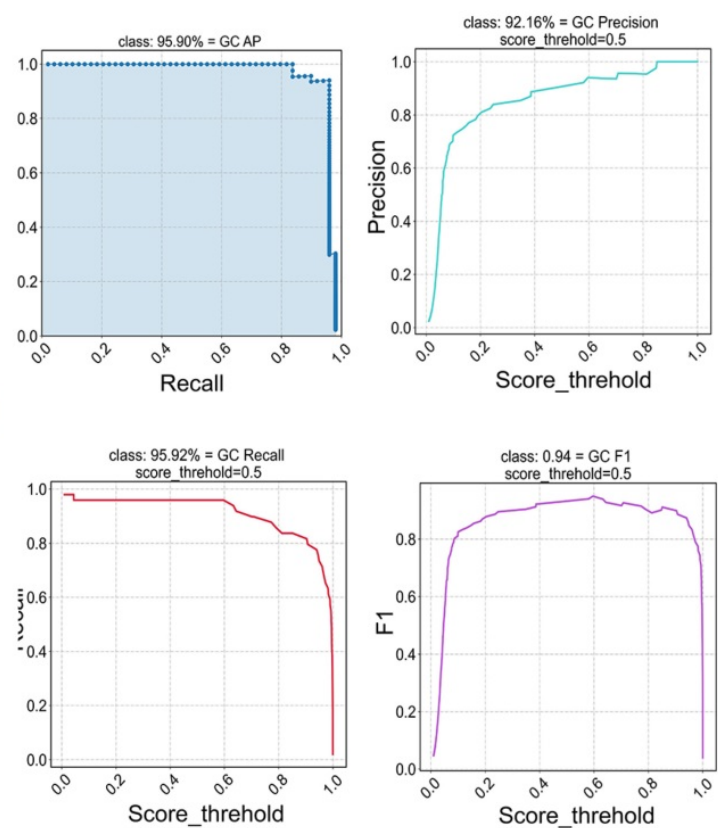

Class GC Confidence 0.78

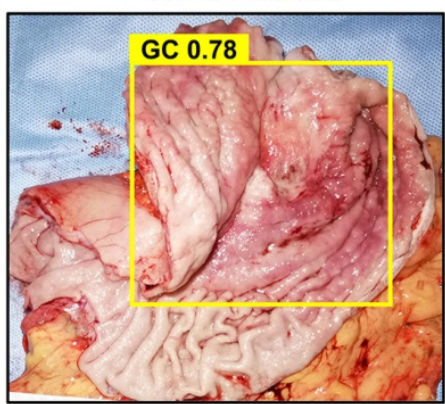

False positive

Confidence 0.68
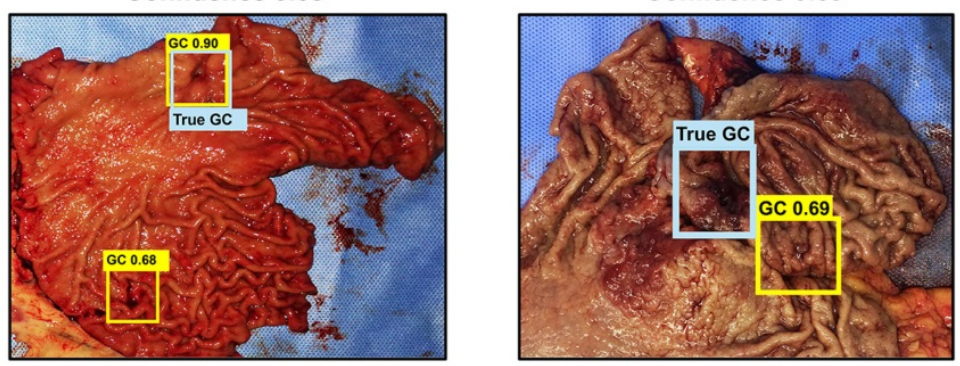

Figure 1. Identification of cancer lesions by the RFB-SSD object detection algorithm. A. The structure of RFB-SSD model. B. The area under the curve of precision and recall (mAP) reached $95.90 \%$ (left up). At 0.5 threshold value, the precision score reached $92.16 \%$ (right up), the recall score $95.92 \%$ (left down) and the F1 score 0.94 (right down). C. True-positive prediction results. All cancer lesions were correctly predicted with high confidence shown on the top of yellow boxes. The predictive confidence of true-positive cases was over $0.7(0.97 \pm 0.07)$. D. False-positive predictive results. The blue labels indicated the true-positive lesions, and the yellow labels represented the false-positive lesions with low confidence $(0.65 \pm 0.05)$.

\section{The performance of semantic segmentation algorithm}

Among the nine selected semantic segmentation algorithms, the ResNet50-PSPNet revealed the highest mIoU (Table 2). So, the ResNet50-PSPNet was used for the subsequent analysis (Figure 2A). After 10-fold cross validation (Table S2), the IoU reached $62.36 \%$
(95\% CI, $60.75 \%-63.97 \%)$, and the mIoU reached $79.26 \%$ (95\% CI, $78.34 \%-80.18 \%$ ). In the test set, the best $\mathrm{IoU}$ and mIoU reached $65.38 \%$ and $80.97 \%$, respectively. The predictive accuracy reached $93.87 \%$ (Figure 2B), and the FNR was 6.13\%. We noticed that the computer might be confused and resulted in false-positive prediction if the mucosa is attached by blood clot or surgical metal clip (Figure 2C). The 
predicting lesions failed in two cases. Both of them were early gastric cancer with superficial depression type, which was rarely learnt by the AI during the training procedure (Figure 2D). In this study, by comparing nine competitive semantic segmentation algorithms, the ResNet50-PSPNet semantic segmentation algorithm performed best with the highest mIoU for positioning cancer lesions.
Table 2. The performance of several semantic segmentation models

\begin{tabular}{llll}
\hline Models & $\begin{array}{l}\text { Feature extraction } \\
\text { network }\end{array}$ & $\begin{array}{l}\text { Semantic } \\
\text { segmentation }\end{array}$ & mIoU \\
\hline MobileNet-PSPNet & MobileNet-v2 & PSPNet & $74.12 \%$ \\
ResNet50-PSPNet & ResNet50 & PSPNet & $79.26 \%$ \\
MobileNet-UNet & MobileNet & UNet & $74.01 \%$ \\
VGG16-UNet & VGG16 & UNet & $73.33 \%$ \\
CENet & ResNet34 & CENet & $44.86 \%$ \\
FCN & VGG16 & FCN & $22.88 \%$ \\
CFPNet & CNN & CFPNet & $55.32 \%$ \\
DCUNet & CNN & DCUNet & $57.15 \%$ \\
ICNet & CNN & ICNet & $48.86 \%$ \\
\hline
\end{tabular}

A

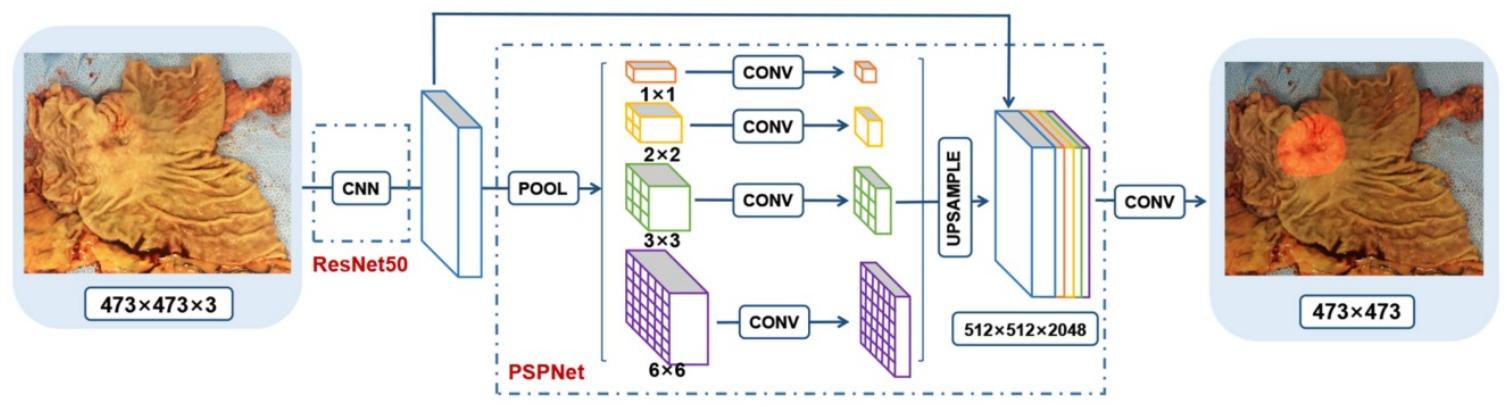

B

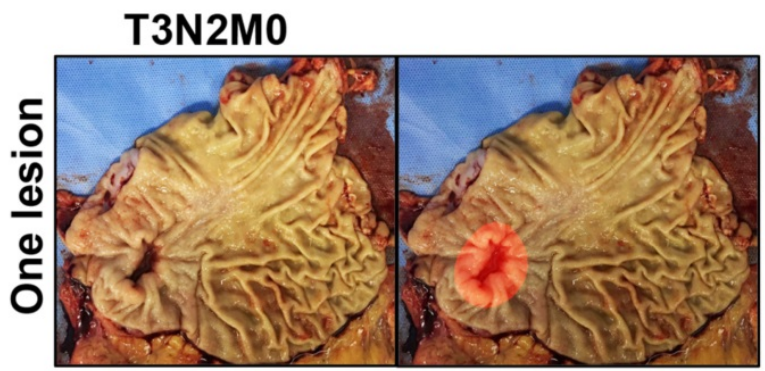

T3N2M0

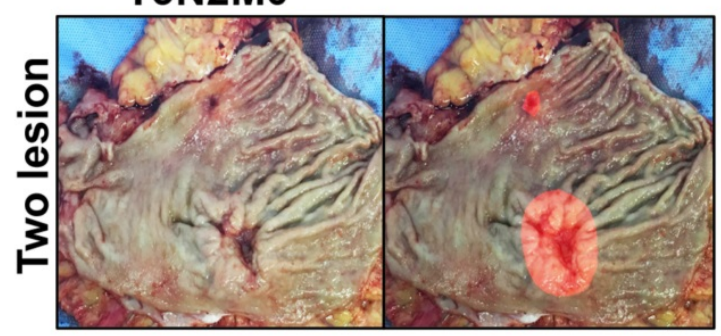

D
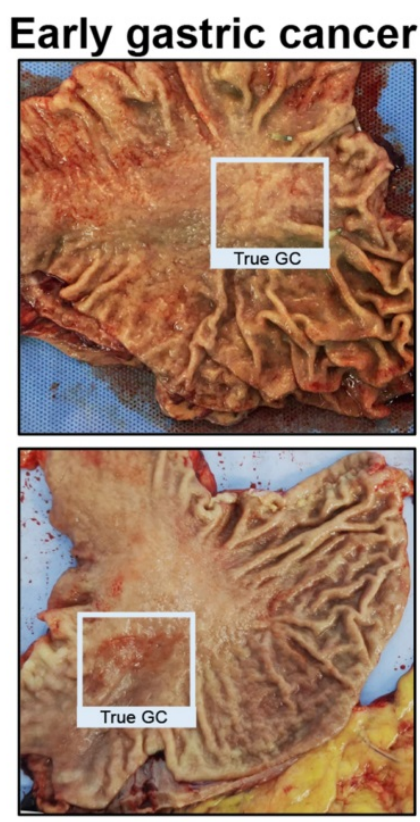

Figure 2. Identification of cancer lesions by the ResNet50-PSPNet semantic segmentation algorithm. A. The network of ResNet50-PSPNet structure. ResNet50 was used as CNN backbone for feature extraction, and PSPNet for semantic segmentation. In the output picture, the predictive region was highlighted as red. B. The true-positive cases were accurately predicted and highlighted as red. C. When gastric mucosa was attached by blood clot (left) or metal clip (right), the computer was confused with false-positive prediction. The red highlighted regions showed the predicted results. The blue boxes labeled true-positive regions, and the yellow boxes labeled the false-positive regions. $\mathbf{D}$. Both failed predictions were early gastric cancers. 
A

\begin{tabular}{|c|cccc|}
\hline & mAP & Precision & Recall & F1 \\
\hline RFB-SSD & $92.60 \%$ & $89.83 \%$ & $92.98 \%$ & 0.91 \\
\hline ResNet50-PSPNet & Accuracy & GC loU & mloU & \\
\hline
\end{tabular}

B
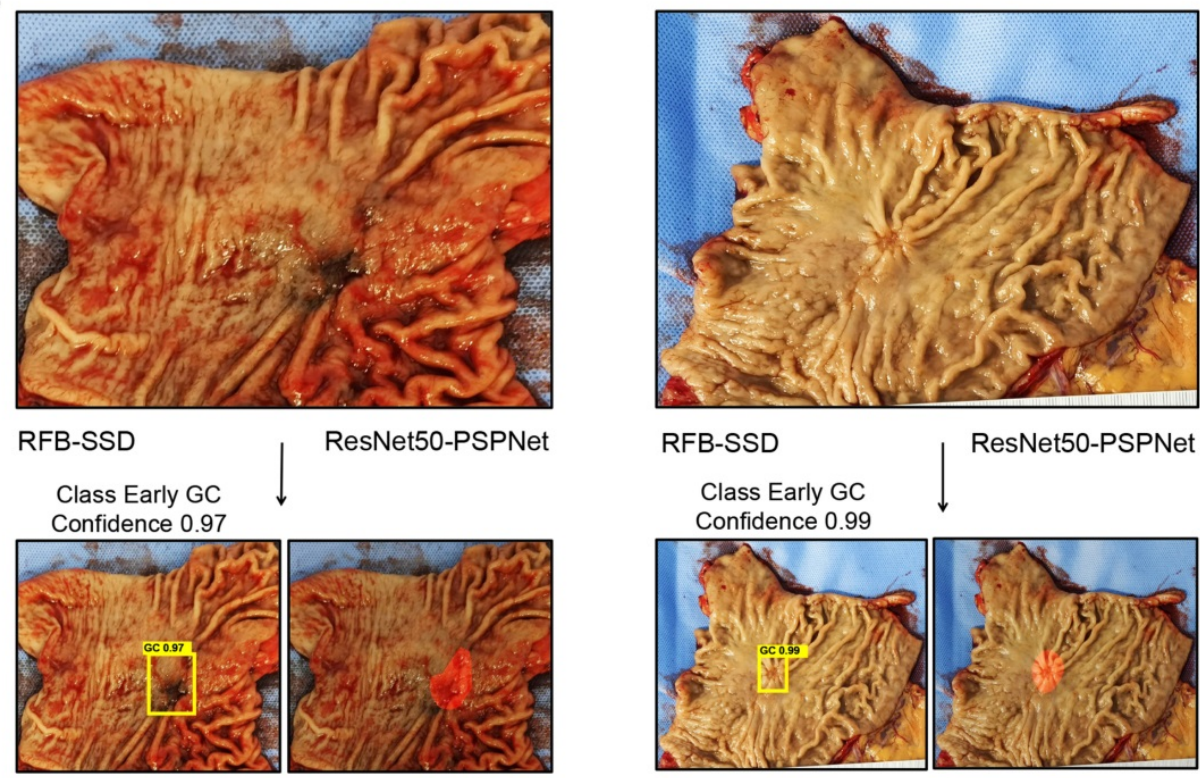

C

D
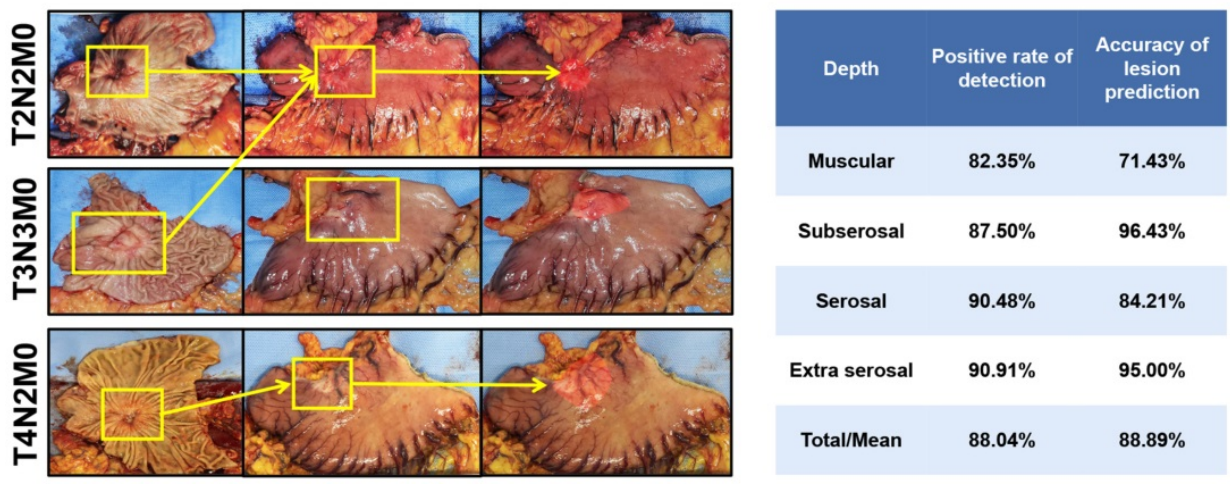

Figure 3. Evaluation of predictive ability by both algorithms on the validation set and the serosal invasion set. A. The performance of RFB-SSD and ResNet50-PSPNet algorithms in the validation set. B. The predictive result of early gastric cancer by the RFB-SSD algorithm and ResNet50-PSPNet algorithm. C. The predictive result of serosa invasion by the ResNet50-PSPNet algorithm analysis. D. The positive rate and accuracy of Al prediction based on cancer invasive depth.

\section{The performance of both algorithms in prospective validation set}

A total of 57 images from 48 patients were included in the prospective validation set. Using the RFB-SSD object detection algorithm, all lesions were detected with $100.00 \%$ accuracy and mAP $92.60 \%$. At the 0.5 threshold, the precision, recall, and F1 were $89.83 \%, 92.98 \%$ and 0.91 respectively (Figure 3A). By the ResNet50-PSPNet semantic segmentation algorithm, the accuracy of locating cancer lesions reached $98.21 \%$ with FNR $1.79 \%$. The IoU and mIoU were $67.03 \%$ and $82.17 \%$ respectively (Figure 3A). Both algorithms revealed good generalization ability for positioning cancer lesions. The time using both
RFB-SSD and ResNet50-PSPNet algorithms for the 57 images was only $8 \mathrm{~s}$ and $7 \mathrm{~s}$ respectively.

We compared the performance of detecting and segmenting lesions by the RFB-SSD and ResNet50-PSPNet models with clinical doctors (Table 3). The diagnostic mAP of RFB-SSD was significantly higher than that of the doctors. The diagnostic mIoU of ResNet50-PSPNet was also significantly higher than that of the doctors. Furthermore, the time for detecting and labeling these 57 images were just several seconds by both RFB-SSD and ResNet50PSPNet algorithms. The labeling time for these 57 images was over 5 minutes for RFB-SSD analysis, and was 18 minutes for ResNet50-PSPNet analysis by the doctors. 
Table 3. The performance comparison between doctors and $\mathrm{Al}$ in validation set

\begin{tabular}{|c|c|c|c|c|c|c|c|c|c|}
\hline & \multicolumn{7}{|c|}{ RFB-SSD } & \multicolumn{2}{|c|}{ ResNet50-PSPNet } \\
\hline & mAP & Precision & Recall & F1 & Accuracy & FNR & Time & $\mathrm{mIoU}$ & Time \\
\hline Doctor 1 & $62.34 \%$ & $68.85 \%$ & $75.00 \%$ & 0.72 & $94.64 \%$ & $5.36 \%$ & $12 \mathrm{~min} 30 \mathrm{~s}$ & $76.67 \%$ & $18 \mathrm{~min} 12 \mathrm{~s}$ \\
\hline Doctor 2 & $47.62 \%$ & $63.16 \%$ & $64.29 \%$ & 0.64 & $91.07 \%$ & $8.93 \%$ & $15 \mathrm{~min} 40 \mathrm{~s}$ & $76.11 \%$ & $20 \mathrm{~min} 5 \mathrm{~s}$ \\
\hline Doctor 3 & $57.14 \%$ & $75.00 \%$ & $75.00 \%$ & 0.75 & $96.40 \%$ & $3.60 \%$ & $6 \mathrm{~min} 41 \mathrm{~s}$ & $81.63 \%$ & $23 \mathrm{~min} 48 \mathrm{~s}$ \\
\hline AI model & $92.60 \%$ & $89.83 \%$ & $92.98 \%$ & 0.91 & $100.00 \%$ & $0.00 \%$ & $8 s$ & $82.17 \%$ & $7 \mathrm{~s}$ \\
\hline
\end{tabular}

We separately analyzed the predictive ability of both algorithms for early gastric cancer (16 images from 14 patients) and advanced gastric cancer (Figure 3B). For early gastric cancer set, the accuracy was $93.75 \%$ and the FNR was $6.25 \%$ with an mAP of $84.17 \%$. At 0.5 threshold, the precision, recall, and F1 were $87.50 \%, 87.50 \%$, and 0.88 , respectively by the RFB-SSD algorithm. Using the ResNet50-PSPNet semantic segmentation algorithm, the predicted accuracy was $81.25 \%$ with IoU and mIoU $37.52 \%$ and $67.99 \%$ respectively. For the advanced gastric cancer set, the mAP was $95.70 \%$. At the 0.5 threshold, the precision, recall, and F1 were $91.40 \%, 95.51 \%$, and 0.93 respectively by the RFB-SSD algorithm. By the ResNet50-PSPNet semantic segmentation algorithm, the IoU and mIoU were $67.11 \%$ and $81.91 \%$ respectively. Therefore, both RFB-SSD and ResNet50PSPNet algorithms revealed good generalization ability and clinical application potential.

\section{The performance of semantic segmentation for serosal invasion}

A total of 92 images of resected gastric specimens were enrolled into serosal invasion analysis by ResNet50-PSPNet algorithm. According to the pathological records, the invasion of muscular layer, subserosal layer, serosal layer, and extra serosa was 17 cases, 32 cases, 21 cases, and 22 cases, respectively. By the ResNet50-PSPNet analysis, the cancer location at serosa of 81 lesions were predicted accurately, and highlighted as red (Figure 3C). The predictive rate was $88.04 \%$. The accuracy of positive cases was $88.89 \%$ and the FNR were $11.11 \%$. The positive rate and accuracy of $\mathrm{AI}$ prediction were different based on their invasive depth (Figure 3D). Therefore, the ResNet50-PSPNet algorithm revealed the high accuracy for predicting serosal invasion.

\section{Performance of semantic segmentation for predicting lymph nodes metastasis}

A total of 556 images from 429 patients (training set plus validation set) were enrolled for prediction of lymph node metastasis. The lymph nodes metastasis was reported in 253 out of 429 cases $(58.97 \%)$ by pathology. By the ResNet50-PSPNet analysis, lymph node metastasis was predicted in 24 cases $(5.59 \%)$ (Figure 4A). Among them, 18 cases were confirmed by pathology $(75 \%)$. The predictive results of primary cancer lesion, the serosa invasion, and the resected lymph nodes in one case were presented in Figure 4B. This result suggested that the ResNet50-PSPNet algorithm revealed limited predicting ability in cancer metastasis to surrounding lymph nodes.

\section{Discussion}

Most medical image analysis by AI was focused on disease classification [25]. Along with the development of AI, several new algorithms revealed powerful functions [5]. For instance, object detection and semantic segmentation algorithms are gradually applied in medical image research including endoscopic images, computed-tomography (CT) images and pathological images [25-33]. However, there is a lack of AI analysis for medical images from macroscopic specimens. Actually, there are a lot of information in macroscopic specimens, such as identification of lesion location (especially for early stage lesion), the cancer classification, surrounding lymph nodes metastasis, and so on. Since our group photographed resected specimens in routine works, we initiated the current study. To our knowledge, this is the first report that AI technology can be used for macroscopic specimen analysis in gastric cancer.

Recently, scientists tried to integrate the traditional $\mathrm{CNN}$ algorithms with object detection and semantic segmentation. The purpose of object detection is to make sure whether there are objects in predefined images. Hirasawa and coworkers utilized the SSD object detection model to accurately recognize early gastric cancer in endoscopic images. The performance of AI detection had higher sensitivity than that of endoscopic physicians [26, 34]. Semantic segmentation is another algorithm in which computer segments images based on the pixels presented in the images. Luo and coworkers utilized DeepLab v3+ semantic segmentation algorithm to precisely segment upper gastrointestinal malignancies in endoscopic images [30]. However, there is no report on using AI technology for recognizing cancer lesions in macroscopic specimens. To find the proper models of the above two algorithms, we screened the performance of 12 object detection models and nine semantic segmentation models. Based on their performance, we proposed 
A
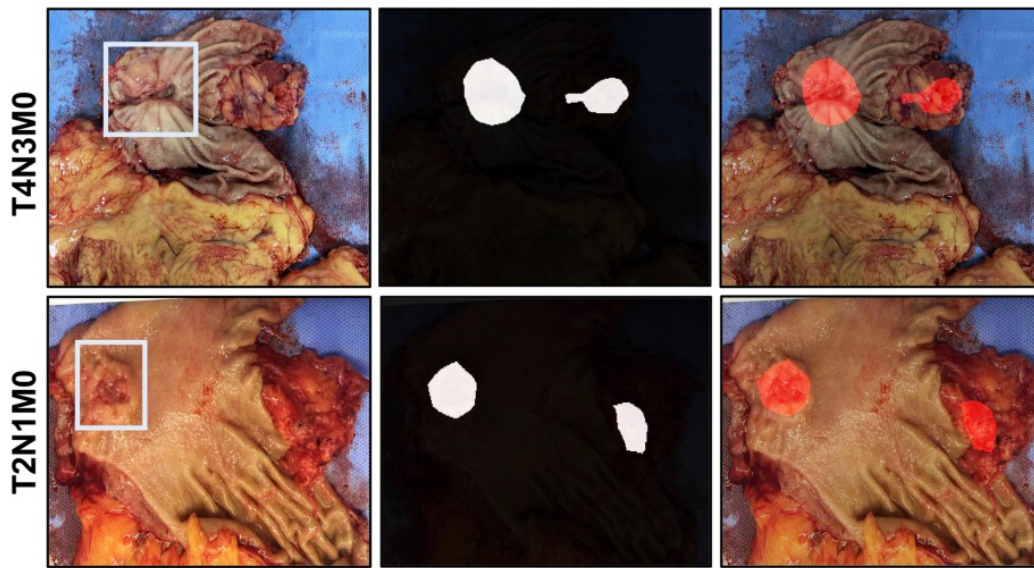

B

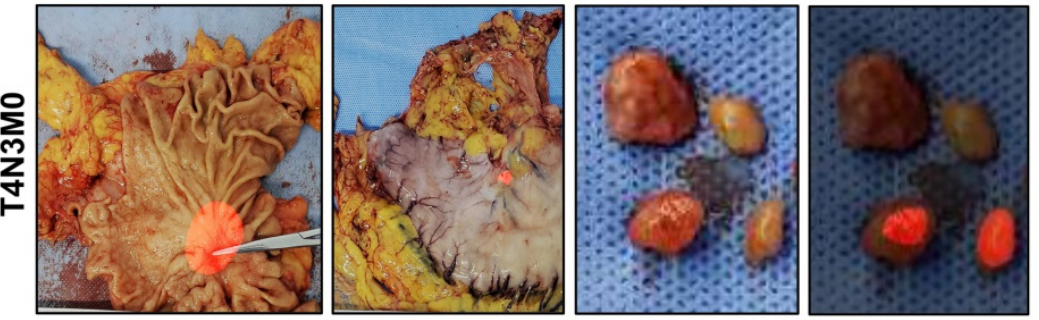

Figure 4. The predictive results of lymph node metastasis by ResNet50-PSPNet algorithm. A. A primary cancer region and a suspected metastatic area of surrounding lymph nodes were highlighted as red. B. The predictive results of primary cancer lesion, the serosa invasion, and the resected lymph nodes in one case were presented. The cancer lesion and the suspected lymph nodes metastasis were highlighted as red.

that the RFB-SSD object detection model and the ResNet50-PSPNet semantic segmentation model are well performed for macroscopic specimen analysis of gastric cancer. Up to date, there is no report utilizing the RFB-SSD object detection model for analyzing medical images. However, PSPNet semantic segmentation model had been used to analyze CT images previously [20]. These two models have been widely used in general images analysis of daily life [22, 35-37].

In the current study, the object detection algorithm provides an accurate recognition of cancer lesions with a high confidence value. For the true-positive cases, all confidence values are 0.7 or above, while the confidence values of false positive cases are lower than 0.7 . The semantic segmentation algorithm provides an automatic segmentation of cancer lesions. Because the large amount of images in our study come from advanced gastric cancer, both RFB-SSD object detection and ResNet50-PSPNet semantic segmentation models performed well in specimens of advanced gastric cancer relative to early gastric cancer. We assumed that we could improve the performance of the models if we collect more images from early gastric cancer and train these models.

In addition, we found that the ResNet50-PSPNet semantic segmentation model can not only recognize and segment primary cancer lesions, but also predict cancer involvement in serosa. This function will guide doctors to avoid damaging cancer lesions when they open the stomach. As to some positively predicted cases with muscular layer invasion, there might be two possible reasons. One is the missed diagnosis of invasive depth by histology. Another is that the invasion of muscular layer by cancer stimulates the cancer-specific fibrosis of serosa. The subtle texture change will be identified by AI. Based on this ability, the ResNet50-PSPNet semantic segmentation model could predict lymph nodes metastasis in a portion of cases. Although the positive predictive rate of lymph nodes is limited, this function of the ResNet50PSPNet semantic segmentation model is worth for further development.

We further compared the performances of the two models with clinical doctors on the parameters of $\mathrm{mAP}$, precision, recall, F1, accuracy, FNR, mIoU and using time. We found that both models performed better than the doctors. Therefore, AI aidedpositioning cancer lesions could improve working efficiency. Since the AI models can not only locate cancer lesions, but also predict serosa involvement as well as lymph nodes metastasis in some cases, AI will have great potential in clinical application. However, both the RFB-SSD and ResNet50-PSPNet models used in the present study are supervised algorithms in AI area. One of the disadvantages of supervised algorithms is the manual annotation, which is expensive and time-consuming. At present, unsupervised AI algorithms are gradually developed, 
which could learn high-quality features without the use of manual labels. Unsupervised learning algorithms have not been widely applied in medical images. Recently, Sari and co-workers have applied unsupervised feature extraction algorithms for colon cancer pathological image classification [38]. We believe that along with the utility of unsupervised algorithms, it not only reduces the laborious manual annotation work, but also finds out subtle texture features that may miss by naked eye.

Our study had some limitations. The performance of both models for detecting early gastric cancer lesions was not satisfactory. Because the morphological difference between early gastric cancer

A

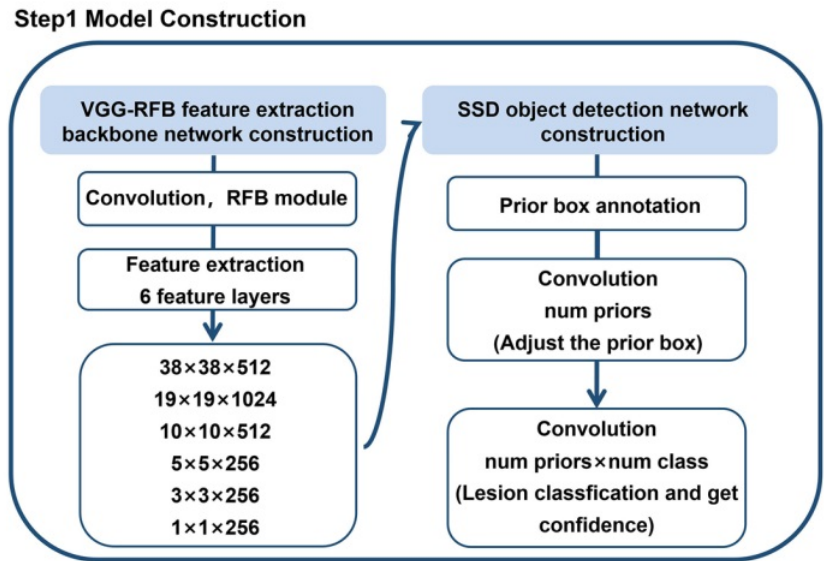

Step2 Image Annotation

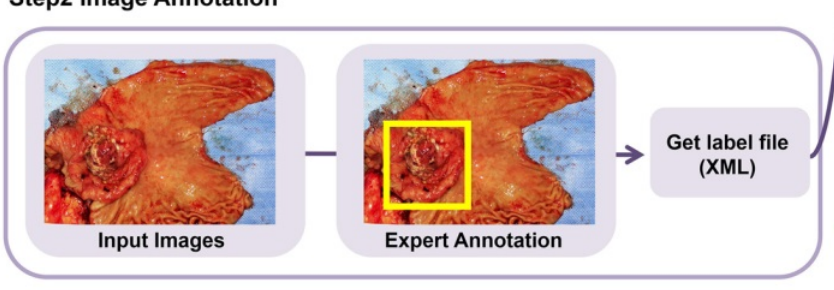

B

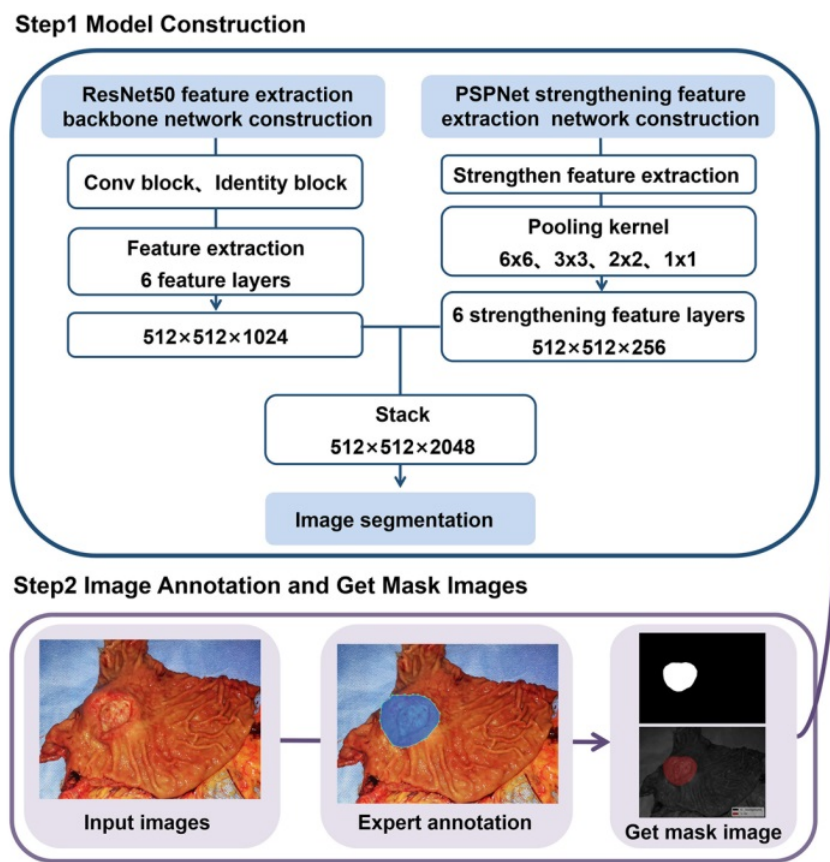

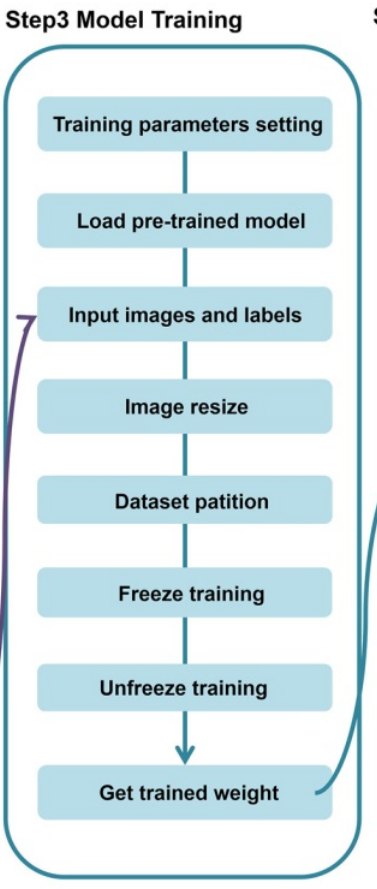
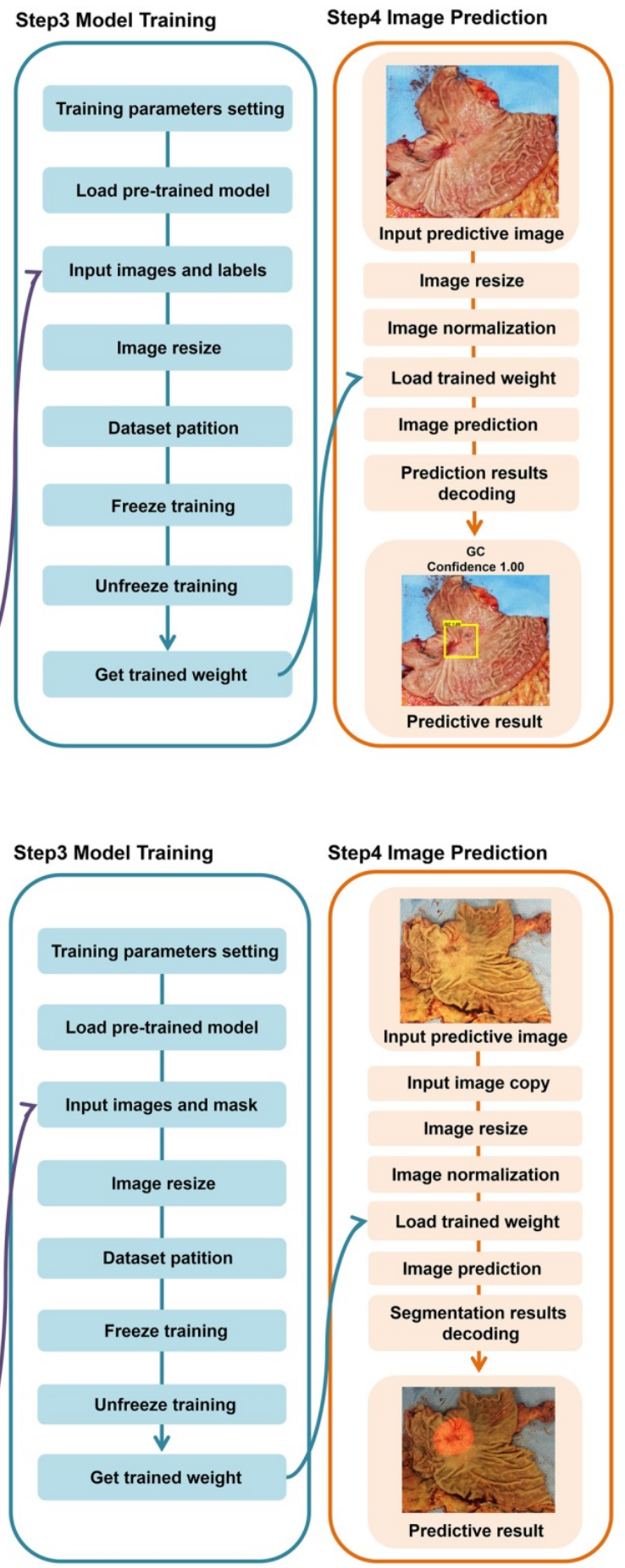

Figure 5. The flow diagrams of RFB-SSD object detection and ResNet50-PSPNet semantic segmentation algorithms. A. The step by step flow diagram of RFB-SSD object detection analysis. B. The step by step flow diagram of ResNet50-PSPNet semantic segmentation analysis. 
with surrounding normal mucosa is not significant, how to improve the performance of AI algorithms for recognizing early gastric cancer is the research direction in the future. We will collect more images from early gastric cancer to train the AI models in our future study. Another limitation is that all images are static. We could not predict the cancer lesions in living status. We will try to develop and integrate the models into the computer of laparoscopic devices for real-time recognition of cancer invasion or lymph nodes metastasis. In addition, the current study is a single center research. A multi-center study to validate the performances of the RFB-SSD and ResNet50-PSPNet models is warranted.

\section{Conclusion}

This study reported a RFB-SSD object detection model (Figure 5A) and a ResNet50-PSPNet semantic segmentation model (Figure 5B) for macroscopic specimens analysis of gastric cancer. Both algorithms effectively identified the subtle features of cancer lesions that may be missed by naked eyes. The AI algorithms could assist doctors quickly locating cancer lesions and improve work efficiency.

\section{Supplementary Material}

Supplementary tables.

http://www.jcancer.org/v12p6473s1.pdf

\section{Acknowledgements}

This project was partially supported by the Shanghai Science and Technology Committee (18411953100 and 20DZ2201900) to YY, the Chinese National Key Program (MOST-2017YFC0908300 and 2016YFC1303200) to YY, the National Natural Science Foundation of China (82072602 and 81772505) to YY, and the Innovation Foundation of Translational Medicine of Shanghai Jiao Tong University School of Medicine (TM202001) to YY.

\section{Competing Interests}

The authors have declared that no competing interest exists.

\section{References}

1. Bray F, Ferlay J, Soerjomataram I, Siegel RL, Torre LA, Jemal A. Global cancer statistics 2018: GLOBOCAN estimates of incidence and mortality worldwide for 36 cancers in 185 countries. CA Cancer J Clin. 2018; 68: 394-424.

2. He J, Baxter SL, Xu J, Xu J, Zhou X, Zhang K. The practical implementation of artificial intelligence technologies in medicine. Nat Med. 2019; 25: 30-6.

3. Lecun Y, Bottou L, Bengio Y, Haffner P. Gradient-based learning applied to document recognition. Proceedings of the IEEE. 1998; 86: 2278-324.

4. Ma B, Guo Y, Hu W, Yuan F, Zhu Z, Yu Y, et al. Artificial Intelligence-Based Multiclass Classification of Benign or Malignant Mucosal Lesions of the Stomach. Front Pharmacol. 2020; 11: 572372.

5. Yang R, Yu Y. Artificial Convolutional Neural Network in Object Detection and Semantic Segmentation for Medical Imaging Analysis. Front Oncol 2021; 11: 638182 .

6. Szegedy C, Liu W, Jia Y, Sermanet P, Reed S, Anguelov D, et al. Going Deeper with Convolutions. IEEE Computer Society. 2014; Boston, MA: 1-9.
7. He K, Zhang X, Ren S, Sun J. Deep Residual Learning for Image Recognition. 2016 IEEE Conference on Computer Vision and Pattern Recognition (CVPR). 2016; Las Vegas, NV: 770-8.

8. Chiu YC, Tsai CY, Ruan MD, Shen GY, Lee TT. Mobilenet-SSDv2: An Improved Object Detection Model for Embedded Systems. 2020 International Conference on System Science and Engineering (ICSSE). 2020; Kagawa, Japan, 2020: 1-5.

9. Tan M, Pang R, Le QV. EfficientDet: Scalable and Efficient Object Detection. 2020 IEEE/CVF Conference on Computer Vision and Pattern Recognition (CVPR). 2020; Seattle, WA, USA: 10778-87.

10. Yadav S, Shukla S. Analysis of k-Fold Cross-Validation over Hold-Out Validation on Colossal Datasets for Quality Classification. 2016 IEEE 6th International Conference on Advanced Computing (IACC). 2016: 78-83.

11. Cho BJ, Bang CS, Park SW, Yang YJ, Seo SI, Lim H, et al. Automated classification of gastric neoplasms in endoscopic images using a convolutional neural network. Endoscopy. 2019; 51: 1121-9.

12. Everingham M, Gool LV, Williams CKI, Winn J, Zisserman A. The Pascal Visual Object Classes (VOC) Challenge. International Journal of Computer Vision. 2010; 88: 303-38

13. Poojary R, Pai A. Comparative Study of Model Optimization Techniques in Fine-Tuned CNN Models. 2019 International Conference on Electrical and Computing Technologies and Applications (ICECTA). 2019; 2019: 1-4.

14. Liu W, Anguelov D, Erhan D, Szegedy C, Reed S, Fu C-Y, et al. SSD: Single Shot MultiBox Detector. Conference on Computer Vision. 2016; Springer, Cham. 2016: 21-37.

15. Redmon J, Divvala S, Girshick R, Farhadi A. You Only Look Once: Unified, Real-Time Object Detection. 2016 IEEE Conference on Computer Vision and Pattern Recognition (CVPR). 2016;: 779-88.

16. Zhuang Z, Liu G, Ding W, Raj ANJ, Qiu S, Guo J, et al. Cardiac VFM visualization and analysis based on YOLO deep learning model and modified 2D continuity equation. Comput Med Imaging Graph. 2020;82: 101732

17. Ren S, He K, Girshick R, Sun J. Faster R-CNN: Towards Real-Time Object Detection with Region Proposal Networks. IEEE Transactions on Pattern Analysis and Machine Intelligence. 2017; 39: 1137-49.

18. Ronneberger O, Fischer P, Brox T. U-Net: Convolutional Networks for Biomedical Image Segmentation. Medical Image Computing and Computer-Assisted Intervention(MICCAI). 2015: 234-41.

19. Chen L-C, Zhu Y, Papandreou G, Schroff F, Adam H. Encoder-Decoder with Atrous Separable Convolution for Semantic Image Segmentation. European Conference on Computer Vision. 2018;: 833-51.

20. Krawczyk Z, Starzyński J. Deep learning approach to creating bone models. 2020 IEEE 21st International Conference on Computational Problems of Electrical Engineering (CPEE). 2020;: 1-4.

21. Abadi M, Barham P, Chen J, Chen Z, Zhang X. TensorFlow: A system for large-scale machine learning. 12th Symposium on Operating Systems Design and Implementation. 2016; 2016: 265-83.

22. Zhu Y, Mu J, Pu H, Shu B. FRFB: Integrate Receptive Field Block Into Feature Fusion Net for Single Shot Multibox Detector. 2018 14th International Conference on Semantics, Knowledge and Grids (SKG). 2018: 173-80.

23. Zhao H, Shi J, Qi X, Wang X, Jia J. Pyramid Scene Parsing Network. 2017 IEEE Conference on Computer Vision and Pattern Recognition (CVPR). 2016; Honolulu, HI, 2017: 6230-9.

24. Abdollahi A, Pradhan B, Alamri A. VNet: An End-to-End Fully Convolutional Neural Network for Road Extraction From High-Resolution Remote Sensing Data. IEEE Access. 2020; 8: 179424-36.

25. Wu L, Zhou W, Wan X, Zhang J, Shen L, Hu S, et al. A deep neural network improves endoscopic detection of early gastric cancer without blind spots. Endoscopy. 2019; 51: 522-31.

26. Hirasawa T, Aoyama K, Tanimoto T, Ishihara S, Shichijo S, Ozawa T, et al. Application of artificial intelligence using a convolutional neural network for detecting gastric cancer in endoscopic images. Gastric Cancer. 2018; 21: 653-60.

27. An P, Yang D, Wang J, Wu L, Zhou J, Zeng Z, et al. A deep learning method for delineating early gastric cancer resection margin under chromoendoscopy and white light endoscopy. Gastric Cancer. 2020; 70: 111-8.

28. Afshari S, BenTaieb A, Hamarneh G. Automatic localization of normal active organs in 3D PET scans. Comput Med Imaging Graph. 2018; 70: 111-8.

29. Liu C, Hu SC, Wang C, Lafata K, Yin FF. Automatic detection of pulmonary nodules on CT images with YOLOv3: development and evaluation using simulated and patient data. Quant Imaging Med Surg. 2020; 10(10): 1917-29.

30. Luo H, Xu G, Li C, He L, Luo L, Wang Z, et al. Real-time artificial intelligence for detection of upper gastrointestinal cancer by endoscopy: a multicentre, case-control, diagnostic study. Lancet Oncol. 2019; 20: 1645-54.

31. Wickstrøm K, Kampffmeyer M, Jenssen R. Uncertainty and interpretability in convolutional neural networks for semantic segmentation of colorectal polyps. Med Image Anal. 2018; 60: 101619.

32. Gao Y, Zhang ZD, Li S, Guo YT, Wu QY, Liu SH, et al. Deep neural network-assisted computed tomography diagnosis of metastatic lymph nodes from gastric cancer. Chin Med J (Engl). 2019; 132: 2804-11.

33. Cai L, Gao J, Zhao D. A review of the application of deep learning in medical image classification and segmentation. Ann Transl Med. 2020; 8(11): 713.

34. Ikenoyama YA-O, Hirasawa TA-O, Ishioka MA-O, Namikawa K, Yoshimizu SA-O, Horiuchi $Y$, et al. Detecting early gastric cancer: Comparison between the diagnostic ability of convolutional neural networks and endoscopists. Digestive Endoscopy 2020;: 1443-661. 
35. Long X, Zhang W, Zhao B. PSPNet-SLAM: A Semantic SLAM Detect Dynamic Object by Pyramid Scene Parsing Network. IEEE Access. 2020; 8: 214685-95.

36. Wang W, Yang J, You X. Combining ElasticFusion with PSPNet for RGB-D Based Indoor Semantic Mapping. 2018 Chinese Automation Congress (CAC); 2018. p. 2996-3001

37. Bui HTT, Le DH, Nguyen TTA, Pham TV. Deep Learning Based Semantic Segmentation for Nighttime Image. 2020 5th International Conference on Green Technology and Sustainable Development (GTSD); 2020. p. 466-71

38. Sari $\mathrm{CT}$, Gunduz-Demir C. Unsupervised Feature Extraction via Deep Learning for Histopathological Classification of Colon Tissue Images. IEEE Trans Med Imaging. 2019; 38: 1139-49. 